\title{
Quantum fluctuations in a continuous vectorial Kerr medium model
}

\author{
Roberta Zambrini, ${ }^{1,2}$ Miguel Hoyuelos, ${ }^{1,2, *}$ Alessandra Gatti, ${ }^{2}$ Pere Colet, ${ }^{1}$ Luigi Lugiato, ${ }^{3}$ and Maxi San Miguel ${ }^{1}$ \\ ${ }^{1}$ Instituto Mediterráneo de Estudios Avanzados, IMEDEA (CSIC-UIB), Campus Universitat Illes Balears, \\ E-07071 Palma de Mallorca, Spain \\ ${ }^{2}$ Dipartimento di Fisica dell'Università di Milano, Istituto Nazionale per la Fisica della Materia, Via Celoria 16, 20133 Milano, Italy \\ ${ }^{3}$ Dipartimento di Scienze CFM, Istituto Nazionale per la Fisica della Materia, Università dell' Insubria, Via Lucini 3,
} 22100 Como, Italy

(Received 28 March 2000; published 1 November 2000)

\begin{abstract}
We consider a model for a Kerr medium in a planar resonator, which takes into account the vectorial character of the radiation field. We analyze the spatial behavior of quantum fluctuations around a steady state, with a roll-pattern configuration in the beam cross section, using a Langevin treatment based on the Wigner representation. The spatial distribution of the quantum fluctuations around the roll pattern is dominated by the neutral (or Goldstone) mode, corresponding to rigid spatial displacements of the pattern. The spatial configuration of the field immediately outside the cavity input-output mirror depends on the time window over which fluctuations are averaged: only when the time window is on the order of the cavity lifetime the output field fluctuations are qualitatively similar to that of the intracavity field. The quantum correlations among the fields in play, as described by the full multimode model, turn out to be in good agreement with those predicted by a simple three-mode model.

PACS number(s): 42.50.Lc, 42.50.Dv, 42.50.Ct, 42.65.Sf
\end{abstract}

\section{INTRODUCTION}

It is by now well known [1] that nonlinear optical patterns display deep quantum aspects that arise essentially from the quantum entanglement or quantum correlation of the spatial modes that generate the pattern [2]. Such quantum aspects are reviewed in Refs. [1b, 3], and arise both above and below the threshold of the instability that creates the pattern. In the above threshold case, the quantum features have been studied almost exclusively for the simplest structure, i.e., the roll (or stripe) pattern, and using three-mode models [4-7] that allow for an analytical calculation of the relevant spectra. A more extended literature concerns the below threshold case, basically devoted to the analysis of the quantum images generated by quantum fluctuations (see, e.g., Refs. [2,8-12]). In these papers the treatment is carried out analytically by taking into account the whole infinite set of transverse cavity modes or, equivalently, by using models in which the radiation field displays full dependence on the continuous spatial variable in the planes transverse with respect to the direction of propagation. Also cavityless configurations have been considered (see, e.g., Refs. [13-18]).

In this paper we will treat the above threshold case using a continuous model, i.e., avoiding any restriction to a reduced number of spatial modes. This can be done by adapting the Langevin treatment of quantum fluctuations and optical patterns introduced in Ref. [10]. A first advantage of this treatment is the possibility of testing the limit of validity of the three-mode model, by using a model that is capable of

\footnotetext{
*Permanent address: Departamento de Física, Facultad de Ciencias Exactas y Naturales, Universidad Nacional de Mar del Plata, Funes 3350, 7600 Mar del Plata, Argentina.
}

describing all the relevant spatial features on any scale of interest.

The main body of our calculations concerns the direct analysis of quantum fluctuations around an inhomogeneous roll-shaped stationary state, by linearization around this steady state. By using the Wigner representation, we describe the dynamics of quantum fluctuations by means of a set of linearized Langevin equations. We also study the behavior of the whole radiation field, without splitting it into the sum of a semiclassical stationary value and of the fluctuations around it. This is obtained by using, again, the Wigner representation. In this case the time evolution equation for the Wigner functional of the radiation field also includes terms with third-order derivatives; however, in the limit of large photon numbers one can argue that these terms can be neglected, and one is left with an approximate Fokker-Planck equation with a positive-definite diffusion matrix, equivalent to a set of classical-looking Langevin equations.

The continuous model offers the possibility of studying single stochastic realizations (snapshots) of the dynamics of the fluctuations of the entire field, while the three-mode model provides a crudely simplified description to this purpose. For example, the three-mode model predicts that, under conditions of translational symmetry, the stripe pattern undergoes random rigid displacements to the left or to the right in the direction orthogonal to the stripes. The pattern maintains a precise orientation, which is determined by an initial fluctuation, but has no definite equilibrium position in the transverse plane, which gives rise to the random rigid displacements, in which the roll position is governed by a diffusive motion ([5], see also Ref. [19]). The result of this process is to wash out the roll pattern itself, if it is observed on a time scale much longer than the diffusion time. The continuous model allows us for investigating the dynamics 
of the roll pattern without introducing the oversimplification of the three-mode theory. However, the drawback is that we have to numerically simulate the Langevin equations, even in the linearized case, because the coefficients of the linearized equations are space dependent since the stationary state around which linearization is performed is inhomogeneous.

Another goal of this paper is to describe the configuration of the field outside the cavity. In all the previous work (see, e.g., Ref. [10]) the Langevin equations were used to simulate the behavior of the intracavity field only. Here we focus, instead, on the output field, immediately beyond the inputoutput mirror of the cavity. The output field arises from the interference of the intracavity field transmitted by the cavity input-output mirror and the input field reflected by the inputoutput mirror. Since the input field is assumed to have whitenoise fluctuations, while the spectral bandwidth (in temporal frequencies) of the intracavity field is determined by the cavity linewidth, the configuration of the output field depends on the time scale over which it is observed.

The system studied in this paper consists of a cavity containing a Kerr medium and driven by a coherent, monochromatic, and linearly polarized plane-wave field. The model $[20,21]$ includes the vectorial character of the field, generalizing the scalar model of Ref. [22]. Our analysis concerns the self-defocusing case, in which one has the formation of a roll pattern orthogonally polarized to the pump at the instability threshold [7,21]. In Ref. [7], by using a three-mode model the anticorrelation between the quantum fluctuations of the intensity of the plane-wave pump and the sum of the intensities of the two tilted waves that generate the pattern was analyzed. More precisely, a quantum-nondemolition (QND) scheme that uses the tilted waves as a meter to measure the intensity fluctuation of the pump, was formulated. In this paper we will compare the prediction of the three-mode model for the QND coefficients, with those of the complete multimode model. We will show the comparison also for the correlation between the two meter beams (i.e., the two twin tilted waves).

The paper is organized as follows: In Sec. II we describe the quantum model and the evolution equation for the quasiprobability distributions of the $c$-number fields associated with the quantum operators. In Sec. III we derive both the nonlinear Langevin equations for the dynamical evolution of the intracavity fields, and the linearized equations for the dynamics of the quantum fluctuations around the inhomogeneous steady state. The result of the numerical integration of these equations is discussed in Sec. IV. The relation between the field inside the cavity and the output field is addressed in Sec. V, where we introduce a way to avoid instantaneous divergences of the output field. In Sec. VI we calculate the quantum correlation between the $x$-polarized homogeneus mode and the $y$-polarized tilted wave modes in the output field as well as the correlation between the two tilted waves and we discuss the applicability of this scheme for QND measurements. We also present the comparison of our results with the simplified three-mode model used in Ref. [7]. In Sec. VII we give some concluding remarks. The paper also includes two appendices, where we give several coefficients corresponding to equations used in the main text.

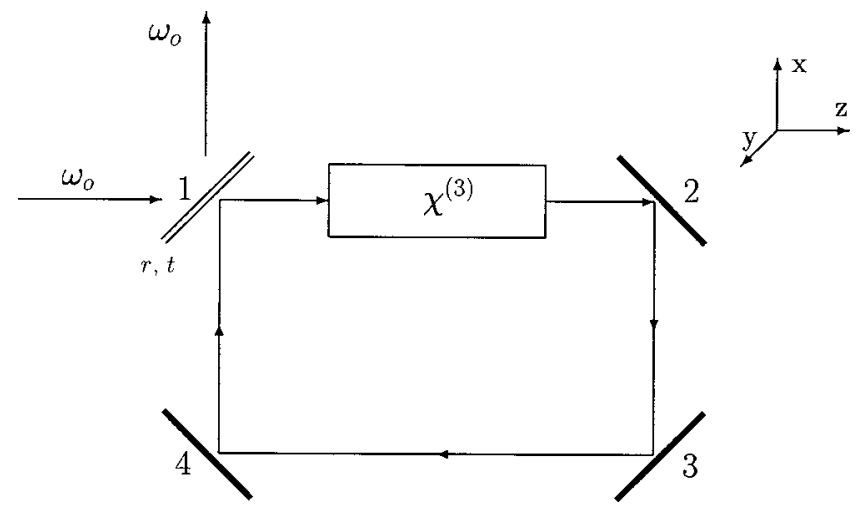

FIG. 1. Ring cavity with a Kerr medium. Mirror 1 partially transmits the input beam with frequency $\omega_{0}$. Mirrors 2, 3, and 4 are completely reflecting.

\section{QUANTUM FORMULATION OF A VECTORIAL KERR RESONATOR}

We consider a one-directional ring cavity (Fig. 1) with four flat mirrors, one of which has a high, but finite reflectivity, and the others are fully reflecting. Inside the cavity is placed a sample of an isotropic Kerr medium, characterized by a third-order susceptibility tensor $\chi_{i j k l}^{(3)}$. The cavity is driven by a coherent, plane-wave, monochromatic and stationary field, with a uniform distribution in the transverse plane and frequency $\omega_{0}$. The input field is linearly polarized, for definiteness along the $x$ direction, so that its circularly polarized components are equal: $E_{0+}=E_{0-}=E_{0}$.

We consider the slowly varying envelope and paraxial approximation, and the cavity mean-field limit [23], that allows to neglect the dependence of the field on the longitudinal coordinate $z$ along the sample. Under these conditions only one longitudinal cavity mode is relevant, precisely the one corresponding to the longitudinal cavity resonance $\omega_{c}$ closest to $\omega_{0}$. We denote by $A_{+}(\vec{x}, t)$ and $A_{-}(\vec{x}, t)$ the intracavity field envelope operators corresponding to the right and left circularly polarized components. These operators depend on the transverse space coordinate $\vec{x}=(x, y)$ and time $t$, and obey standard equal-time commutation relations,

$$
\left[A_{i}(\vec{x}, t), A_{j}^{\dagger}\left(\vec{x}^{\prime}, t\right)\right]=\delta_{i j} \delta\left(\vec{x}-\vec{x}^{\prime}\right),
$$

where the indexes $i, j$ stand for,+- . By adopting a picture in which the fast oscillation at the carrier frequency $\omega_{0}$ is eliminated, the reversible part of the dynamics of the intracavity field is described by the following Hamiltonian [7], that represents a generalization to the vectorial case of the one introduced in Ref. [4] for a scalar Kerr medium:

$$
H=H_{0}+H_{\text {ext }}+H_{\text {int }} .
$$

$H_{0}$ describes the free propagation of the intracavity field in the paraxial approximation: 


$$
\begin{aligned}
H_{0}= & \hbar K \int d^{2} \vec{x}\left[A_{+}^{\dagger}(\vec{x})\left(\eta \theta-a \nabla^{2}\right) A_{+}(\vec{x})\right. \\
& \left.+A_{-}^{\dagger}(\vec{x})\left(\eta \theta-a \nabla^{2}\right) A_{-}(\vec{x})\right],
\end{aligned}
$$

where $K=c T /(2 \mathcal{L})$ is the cavity linewidth, with $c$ being the speed of light, $T$ the transmittivity coefficient of the inputoutput mirror, and $\mathcal{L}$ the total cavity length; $\eta \theta$ is the cavity detuning parameter; $\eta$ is the sign of the Kerr nonlinearity ( $\eta=1$ for the self-focusing case and $\eta=-1$ for the selfdefocusing case); $\nabla^{2}$ is the two-dimensional transverse Laplacian that models the effect of diffraction in the paraxial approximation; the parameter $a$ has the dimension of an area, so that $l_{D}=\sqrt{a} \approx \sqrt{\mathcal{L} \lambda /(2 \pi T)}$, with $\lambda$ being the wavelength, defines the length scale for transverse pattern formation.

$H_{e x t}$ models the coherent pumping by a classical planewave driving field of amplitude $E_{0}$ :

$$
H_{\text {ext }}=i \hbar K E_{0} \int d^{2} \vec{x}\left[A_{+}^{\dagger}(\vec{x})-A_{+}(\vec{x})+A_{-}^{\dagger}(\vec{x})-A_{-}(\vec{x})\right],
$$

where, without any loss of generality, $E_{0}$ has been taken real.

$H_{\text {int }}$ is the interaction Hamiltonian that describes the coupling due to the Kerr nonlinearity:

$$
\begin{aligned}
H_{i n t}= & -\eta \hbar K g \int d^{2} \vec{x}\left\{\frac{\alpha}{2}\left[A_{+}^{\dagger}(\vec{x}) A_{+}^{2}(\vec{x})+A_{-}^{\dagger}(\vec{x}) A_{-}^{2}(\vec{x})\right]\right. \\
& \left.+\beta\left[A_{+}^{\dagger}(\vec{x}) A_{-}^{\dagger}(\vec{x}) A_{+}(\vec{x}) A_{-}(\vec{x})\right]\right\},
\end{aligned}
$$

where the coupling constant $g$ is related to the element $\chi_{1111}^{(3)}$ of the susceptibility tensor. Constants $\alpha$ and $\beta$ are also related to the susceptibility tensor components [24]. For an isotropic medium they satisfy $\alpha+\beta=2$. We will use typical values for a liquid Kerr medium: $\alpha=1 / 4$ and $\beta=7 / 4$ $[20,21,25]$.

The intracavity dynamics are described by a master equation for the reduced density operator $\rho$ of the system:

$$
\frac{\partial \rho}{\partial t}=\frac{1}{i \hbar}[H, \rho]+\Lambda \rho .
$$

The Liouvillian $\Lambda$ accounts for dissipation through the partially reflecting mirror, and is given by

$$
\Lambda \rho=K \sum_{i=+,-} \int d^{2} \vec{x}\left\{\left[A_{i}(\vec{x}), \rho A_{i}^{\dagger}(\vec{x})\right]+\left[A_{i}(\vec{x}) \rho, A_{i}^{\dagger}(\vec{x})\right]\right\} .
$$

Using the quantum-to-classical correspondence [26,27], Eq. (6) can be converted into an equation of motion for quasiprobability distributions in the classical phase space of the system; these are functionals of the $c$-number fields $\alpha_{ \pm}(\vec{x})$ associated with the operators $A_{ \pm}(\vec{x})$. In order to obtain this equation, it is enough to formally substitute products of field operators and the density operator by suitable operations over the distribution functionals according to the following scheme of correspondence relations [10]:

$$
\rho A_{ \pm}^{\dagger}(\vec{x}) \Leftrightarrow\left(\alpha_{ \pm}^{*}(\vec{x})+\frac{1-s}{2} \frac{\delta}{\delta \alpha_{ \pm}(\vec{x})}\right) W_{s}\left(\alpha_{+}, \alpha_{+}^{*}, \alpha_{-}, \alpha_{-}^{*}\right),
$$

$$
\begin{aligned}
& A_{ \pm}^{\dagger}(\vec{x}) \rho \Leftrightarrow\left(\alpha_{ \pm}^{*}(\vec{x})-\frac{1+s}{2} \frac{\delta}{\delta \alpha_{ \pm}(\vec{x})}\right) W_{s}\left(\alpha_{+}, \alpha_{+}^{*}, \alpha_{-}, \alpha_{-}^{*}\right), \\
& \rho A_{ \pm}(\vec{x}) \Leftrightarrow\left(\alpha_{ \pm}(\vec{x})-\frac{1+s}{2} \frac{\delta}{\delta \alpha_{ \pm}^{*}(\vec{x})}\right) W_{s}\left(\alpha_{+}, \alpha_{+}^{*}, \alpha_{-}, \alpha_{-}^{*}\right),
\end{aligned}
$$$$
A_{ \pm}(\vec{x}) \rho \Leftrightarrow\left(\alpha_{ \pm}(\vec{x})+\frac{1-s}{2} \frac{\delta}{\delta \alpha_{ \pm}^{*}(\vec{x})}\right) W_{s}\left(\alpha_{+}, \alpha_{+}^{*}, \alpha_{-}, \alpha_{-}^{*}\right) .
$$

Here the parameter $1 \geqslant s \geqslant-1$ defines the choice of the representation, namely, $s=+1$ corresponds to the GlauberSudarshan $P$ distribution; $s=0$ to the Wigner $W$ distribution, and $s=-1$ to the Husimi $Q$ distribution. The symbols $\delta / \delta \alpha_{ \pm}(\vec{x})$ stand for functional derivatives.

The evolution equation for the quasiprobability distributions is given by

$$
\begin{aligned}
\frac{\partial W_{s}\left(z_{1}, z_{2}, z_{3}, z_{4}\right)}{\partial t}= & \int d^{2} \vec{x} K\left[-\sum_{i=1}^{4}\left(\frac{\delta}{\delta z_{i}(\vec{x})} Q_{i}\right)+\frac{1}{2} \int d^{2} \vec{x}^{\prime} \sum_{i, j=1}^{4} \frac{\delta^{2}}{\delta z_{i}(\vec{x}) \delta z_{j}\left(\vec{x}^{\prime}\right)} D_{i j}\left(\vec{x}, \vec{x}^{\prime}\right)\right. \\
& \left.+\frac{1}{6} \int d^{2} \vec{x}^{\prime} \int d^{2} \vec{x}^{\prime \prime} \sum_{i, j, l=1}^{4} \frac{\delta^{3}}{\delta z_{i}(\vec{x}) \delta z_{j}\left(\vec{x}^{\prime}\right) \delta z_{l}\left(\vec{x}^{\prime \prime}\right)} T_{i j l}\left(\vec{x}, \vec{x}^{\prime}, \vec{x}^{\prime \prime}\right)\right] W_{s}\left(z_{1}, z_{2}, z_{3}, z_{4}\right),
\end{aligned}
$$

where $\left(z_{1}, z_{2}, z_{3}, z_{4}\right)=\left(\alpha_{+}, \alpha_{+}^{*}, \alpha_{-}, \alpha_{-}^{*}\right)$. The coefficients $Q_{i}, D_{i j}$, and $T_{i j l}$ are given in Appendix A. 


\section{DERIVATION OF THE LANGEVIN EQUATIONS}

The time evolution equation (9) derived in the previous section is of little practical use, both from an analytical and computational point of view; for example in the $P$ representation $(s=-1)$ the diffusion matrix is not positive definite, while in the Wigner representation $(s=0)$ the third-order derivative terms do not vanish. In order to derive a more manageable equation we have to introduce some approximation. The standard scheme (see, e.g., Ref. [27], Sec. IV) involves an expansion of Eq. (9) in terms of a parameter measuring the inverse system size. In this way one obtains at the same time the time evolution equations for classical field amplitudes, and those describing the dynamics of fluctuations around the classical macroscopic fields. This is equivalent to the linearization procedure outlined in the second part of this section.

In our model the parameter $g^{-1}$ plays the role of a scaling factor for the photon number; precisely $n_{s}=a g^{-1}$ represents the intracavity saturation photon number on the characteristic area $a$ in the transverse plane, and typically is a very large number. By reformulating Eq. (9) in terms of scaled fields, $\tilde{z_{i}}=\sqrt{g} z_{i}$, it is readily seen that the second-order derivative terms scale as $g$, while the third-order derivative terms scale as $g^{2}$ (the first-order derivative has terms of order $g^{0}$ and $g$ ). In the case of the Wigner representation, it is possible to neglect the third-order derivatives (order $g^{2}$ ), resulting in an Fokker-Planck equation that has a positive definite diffusion matrix [28]. From the Fokker-Planck equation we can obtain a equivalent set of Langevin equations. Once we have the Langevin equation a further approximation is possible: the drift $Q_{1}$, in terms of the scaled fields, takes the form $Q_{1}=\left[-(1+i \eta \theta)+i g \eta(\alpha+\beta / 2)(s-1)+i a \nabla^{2}\right] z_{1}+E_{o}$ $+i \eta\left[\alpha z_{1} z_{2}+\beta z_{3} z_{4}\right] z_{1}$ where the term $\operatorname{ig} \eta(\alpha+\beta / 2)(s$ -1 ) is of higher order in $g$ and can be neglected. Proceeding in a similar way for all the drift terms, the set of Langevin equations, where we keep terms up to order $g^{1 / 2}$ (the noise terms), finally read:

$$
\begin{aligned}
\frac{\partial \alpha_{ \pm}}{\partial t}= & -(1+i \eta \theta) \alpha_{ \pm}+i \nabla^{2} \alpha_{ \pm}+E_{0}+i \eta\left[\alpha\left|\alpha_{ \pm}\right|^{2}\right. \\
& \left.+\beta\left|\alpha_{\mp}\right|^{2}\right] \alpha_{ \pm}+\sqrt{2} \xi_{ \pm}(\vec{x}, t),
\end{aligned}
$$

where, in order to simplify the notation, we have introduced the scaled variables:

$$
\begin{gathered}
\tilde{x}=x / \sqrt{a}, \\
\tilde{t}=K t, \\
\widetilde{E}_{0}=\sqrt{g} E_{0}, \\
\tilde{\alpha}_{j}=\sqrt{g} \alpha_{j},
\end{gathered}
$$

and omitted the tildes. Here $\xi_{ \pm}(\vec{x}, t)$ are complex Gaussian random variables of zero mean and variance given by

$$
\begin{gathered}
\left\langle\xi_{i}(\vec{x}, t) \xi_{j}^{*}\left(\vec{x}^{\prime}, t^{\prime}\right)\right\rangle=\frac{1}{2 n_{s}} \delta_{i j} \delta\left(\vec{x}-\vec{x}^{\prime}\right) \delta\left(t-t^{\prime}\right), \\
\left\langle\xi_{i}(\vec{x}, t) \xi_{j}\left(\vec{x}^{\prime}, t^{\prime}\right)\right\rangle=0 .
\end{gathered}
$$

By letting $n_{s} \rightarrow \infty$, and hence dropping the stochastic noise terms $\xi_{i}$, one recovers the classical time evolution equations of the model $[20,21]$, for the macroscopic fields $E_{ \pm}(\vec{x}, t)$ :

$$
\begin{aligned}
\frac{\partial E_{ \pm}}{\partial t}= & -(1+i \eta \theta) E_{ \pm}+i \nabla^{2} E_{ \pm}+E_{0} \\
& +i \eta\left[\alpha\left|E_{ \pm}\right|^{2}+\beta\left|E_{\mp}\right|^{2}\right] E_{ \pm} .
\end{aligned}
$$

Thus Eq. (10) can be as well interpreted as the classical nonlinear equations of the model, with a Gaussian noise term added. The derivation of these equations from a quantum model adds to this picture the amplitude of the Gaussian noise, and allows us to interpret it as vacuum fluctuations entering the input-output cavity mirror.

In the rest of this section we will be interested in describing the dynamics of small quantum fluctuations around the classical mean value, whose dynamics are described by Eq. (14). Rather than deriving this dynamics from the inverse system size expansion of Eq. (9), we will use an equivalent procedure, which amounts to expanding the master equation (6) in a power series of fluctuation operators. To this purpose we separate the field operators into two parts,

$$
A_{ \pm}(\vec{x}, t)=F_{ \pm}(\vec{x})+\delta A_{ \pm}(\vec{x}, t)
$$

where $F_{ \pm}(\vec{x})$ are $c$-number fields, representing macroscopic, classical stationary fields, and $\delta A_{ \pm}(\vec{x}, t)$ are fluctuation operators. Next, we expand both the Hamiltonian (2) and the Liouvillian operator (7) in power series of the fluctuation operators $\delta A_{ \pm}(\vec{x}, t)$ :

$$
\begin{gathered}
H=H^{(0)}+H^{(1)}+H^{(2)}+\cdots, \\
\Lambda=\Lambda^{(0)}+\Lambda^{(1)}+\Lambda^{(2)} .
\end{gathered}
$$

The zero-order terms $H^{(0)}, \Lambda^{(0)}$ do not give any contribution to the dynamics; the first-order contributions give rise in the master equation to terms that vanish identically when the $c$-number fields $F_{ \pm}(\vec{x})$ are taken as the steady-state solution of the classical time evolution equations (14).

The second-order term of the Hamiltonian is explicitly given by

$$
H_{0}^{(2)}=\hbar K \int d^{2} \vec{x} \sum_{i=+,-} \delta A_{i}^{\dagger}(\vec{x})\left(\eta \theta-a \nabla^{2}\right) \delta A_{i}(\vec{x}),
$$

$$
H_{\text {ext }}^{(2)}=0 \text {, }
$$




$$
\begin{aligned}
H_{i n t}^{(2)}= & -\eta \hbar K g \int d^{2} \vec{x} \frac{\alpha}{2} \sum_{i=+,-}\left[\delta A_{i}^{2}(\vec{x}) F_{i}^{* 2}(\vec{x})\right. \\
& \left.+\delta A_{i}^{\dagger 2}(\vec{x}) F_{i}^{2}(\vec{x})+4\left|F_{i}(\vec{x})\right|^{2} \delta A_{i}^{\dagger}(\vec{x}) \delta A_{i}(\vec{x})\right] \\
& +\beta\left[F_{+}(\vec{x}) F_{-}(\vec{x}) \delta A_{+}^{\dagger}(\vec{x}) \delta A_{-}^{\dagger}(\vec{x})\right. \\
& \left.+F_{+}(\vec{x}) F_{-}^{*}(\vec{x}) \delta A_{+}^{\dagger}(\vec{x}) \delta A_{-}(\vec{x})+\text { H.c. }\right] \\
& +\beta\left[\left|F_{+}(\vec{x})\right|^{2} \delta A_{-}^{\dagger}(\vec{x}) \delta A_{-}(\vec{x})\right. \\
& \left.+\left|F_{-}(\vec{x})\right|^{2} \delta A_{+}^{\dagger}(\vec{x}) \delta A_{+}(\vec{x})\right] .
\end{aligned}
$$

Higher order terms in the Hamiltonian are neglected, on the basis of a small quantum noise approximation, which is valid for a macroscopic system, i.e., for large saturation photon number $n_{s}$, not too close to critical points. The expression for the Liouvillian $\Lambda^{(2)}$ is obtained by simply replacing $A_{ \pm}(\vec{x})$ by $\delta A_{ \pm}(\vec{x})$ in Eq. (7).

Next we apply to the approximated master equation the correspondence relations (8), with the field operators replaced by the fluctuation operators. In the Wigner representation we obtain the following Fokker-Planck equation:

$$
\frac{\partial W_{0}\left(z_{1}, z_{2}, z_{3}, z_{4}\right)}{\partial t}=\int d^{2} \vec{x}\left[-\sum_{i=1}^{4}\left(\frac{\delta}{\delta z_{i}(\vec{x})} Q_{i}\right)+\frac{1}{2} \int d^{2} \vec{x}^{\prime} \sum_{i, j=1}^{4} \frac{\delta^{2}}{\delta z_{i}(\vec{x}) \delta z_{j}\left(\vec{x}^{\prime}\right)} D_{i j}\left(\vec{x}, \vec{x}^{\prime}\right)\right] W_{0}\left(z_{1}, z_{2}, z_{3}, z_{4}\right)
$$

where now $\left(z_{1}, z_{2}, z_{3}, z_{4}\right)=\left(\Delta \alpha_{+}, \Delta \alpha_{+}^{*}, \Delta \alpha_{-}, \Delta \alpha_{-}^{*}\right)$ are the $c$-number fields corresponding to the fluctuation operators. The drift and diffusion terms $\left(Q_{i}\right.$ and $\left.D_{i j}\right)$ are given in Appendix B. As it is well-known, for a quadratic Hamiltonian, the diffusion matrix of the Fokker-Planck equation in the Wigner representation is positive definite. This condition is not necessarily fulfilled in other representations. A positive definite diffusion matrix allows us to interpret $\Delta \alpha_{+}$and $\Delta \alpha_{-}$as classical stochastic processes, described by a set of Langevin equations, equivalent to Eq. (18), that are given by

$$
\begin{aligned}
\frac{\partial \Delta \alpha_{ \pm}(\vec{x}, t)}{\partial t}= & \left\{-(1+i \eta \theta)+i \nabla^{2}+i \eta\left[2 \alpha\left|F_{ \pm}(\vec{x})\right|^{2}\right.\right. \\
& \left.\left.+\beta\left|F_{\mp}(\vec{x})\right|^{2}\right]\right\} \Delta \alpha_{ \pm}(\vec{x}, t) \\
& +i \eta\left[\alpha F_{ \pm}^{2}(\vec{x}) \Delta \alpha_{ \pm}^{*}(\vec{x}, t)\right. \\
& +\beta F_{ \pm}(\vec{x}) F_{+}^{*}(\vec{x}) \Delta \alpha_{\mp}(\vec{x}, t) \\
& \left.+\beta F_{ \pm}(\vec{x}) F_{\mp}(\vec{x}) \Delta \alpha_{+}^{*}(\vec{x}, t)\right]+\sqrt{2} \Delta \alpha_{ \pm}^{i n}(\vec{x}, t) .
\end{aligned}
$$

In this equation the scaling is the same as in Eq. (11), for what concerns time, space, and the stationary classical fields while fluctuations are scaled with $\sqrt{a}$.

The stochastic terms $\Delta \alpha_{ \pm}^{i n}(\vec{x}, t)$ can again be interpreted as vacuum fluctuations entering the cavity through the coupling mirror [29], and are described by Gaussian white noise, with zero average and correlations given by

$$
\begin{gathered}
\left\langle\Delta \alpha_{i}^{i n}(\vec{x}, t) \Delta \alpha_{j}^{* i n}\left(\vec{x}^{\prime}, t^{\prime}\right)\right\rangle=\frac{1}{2} \delta_{i j} \delta\left(\vec{x}-\vec{x}^{\prime}\right) \delta\left(t-t^{\prime}\right), \\
\left\langle\Delta \alpha_{i}^{i n}(\vec{x}, t) \Delta \alpha_{j}^{i n}\left(\vec{x}^{\prime}, t^{\prime}\right)\right\rangle=0,
\end{gathered}
$$

where the subindices $i, j$ stand for the circularly polarized components,+- .

\section{INTEGRATION OF LANGEVIN EQUATIONS}

We consider the situation described by the classical equations (14) in which an instability leads to the formation of a stripe pattern in the transverse cross section of the intracavity field. For a linearly polarized input $E_{0}$, equations (14) admit a stationary homogeneous solution, linearly polarized in the same direction than the pump, and given by

$$
E_{+}^{s t}=E_{-}^{s t}=E^{s t}, \quad\left|E_{0}\right|^{2}=\left|E^{s t}\right|^{2}\left[1+\left(2\left|E^{s t}\right|^{2}-\theta\right)^{2}\right] .
$$

In the self-defocusing case $\eta=-1$, the steady state (21) becomes unstable when the pump intensity is increased to a value such that $\left|E^{s t}\right|^{2}>\left|E_{c}^{s t}\right|^{2}=1 /(\beta-\alpha)=2 / 3[20,21]$. For an $x$-polarized pump field, and immediately above this instability threshold, a $y$-polarized stripe pattern emerges, characterized by the critical transverse wave vector

$$
k_{c}=\sqrt{\theta-\alpha /(1-\alpha)},
$$

while the $x$ component remains basically homogeneous [30]. This is usually called a polarization pattern since it emerges as a consequence of a polarization instability.

The classical fields $F_{ \pm}(\vec{x})$ describing these stripe patterns can be obtained by numerical integration [31] of the dynamical equation (14). The orientation of the stripes is selected by the initial condition. We choose an initial condition that favors the formation of vertical stripes. These fields obtained numerically enter as space-dependent coefficients in the linearized Langevin equations (19). Fluctuations are calculated from these stochastic equations, which are numerically integrated using a pseudospectral method in Fourier space with periodic boundary conditions. The method is first-order accurate in time [32]. Note that Eq. (19) describes the linearized fluctuations around a fixed stationary solution.

A main qualitative aspect of the fluctuations calculated numerically in this way is the following. In a single stochastic realization of equations (19), the fluctuations of the $x$-polarized field component appear homogeneously distrib- 


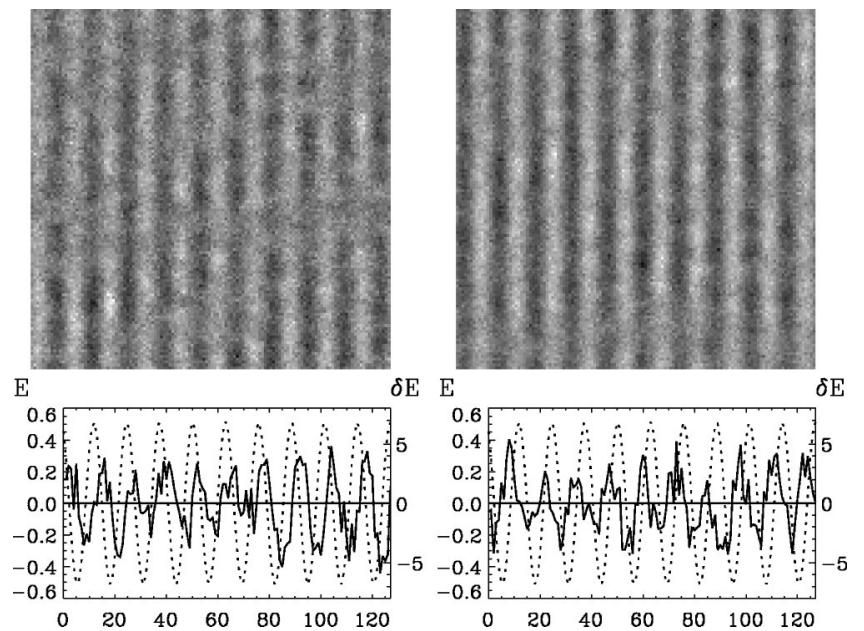

FIG. 2. Upper plots: distribution in the transverse plane of the real part of the $y$-polarized fluctuations for two different realizations in a stationary regime $\left(K t \sim 10^{4}\right)$. For these two realizations the patterns of fluctuations are shifted half of the wavelength one with respect to the other. Bottom plots: The solid line shows the cross section of the real part of the scaled $y$-polarized fluctuations at $y$ $=10$. For comparison, the dotted line shows the real part of the scaled $y$-polarized stationary solution. We note that, as explained in the text, the scaling of the stationary solution and the fluctuations is different. The transverse system size is in scaled units $L=128$ $\times \Delta x$ where the integration discretization is $\Delta x=0.6012$. We have taken $\theta=1, E_{0}=0.919=1.07 E_{0}^{c}$.

uted in space; this follows from the spatial homogeneity of the $x$-polarized component of the stationary classical solution. However, fluctuations in the $y$-polarized component tend to be distributed in space with a stripe structure, similar to that of the corresponding steady-state solution, but shifted to the left or to the right by a quarter-period, as shown in Fig. 2.

The spatial structure of the field fluctuations can be understood in terms of a Goldstone mode [33] as we explain in the following. Very generally, consider a set of fields $\psi_{i}(\vec{x}, t)$ that obey dynamical equations of the form

$$
\frac{\partial \psi_{i}(\vec{x}, t)}{\partial t}=F_{i}\left(\nabla, \psi_{1}, \ldots, \psi_{N}\right),
$$

where $F_{i}$ is a general functional of $\nabla$ and the fields $\psi_{i}(\vec{x}, t)$, and such that they admit a stationary configuration $\psi_{i}^{0}(\vec{x})$. The linear analysis of fluctuations around this stationary configuration is made by calculating the eigenvalues of the matrix operator

$$
M_{l j}=\left[\frac{\delta F_{l}}{\delta \psi_{j}}\right]_{\psi_{i}=\psi_{i}^{0}} .
$$

If the system is translationally invariant, $\psi_{i}^{0}\left(\vec{x}+\vec{x}_{0}\right)$ is also a stationary configuration for any fixed $\vec{x}_{0}$. From this condition, and in a system with spatial dimensionality $D$, it follows immediately that the $D$ independent components of the

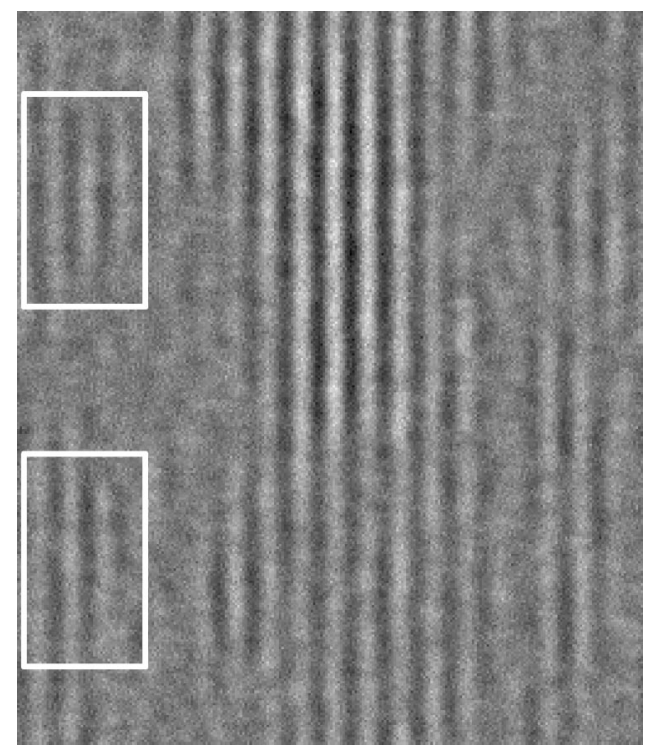

FIG. 3. Fluctuations ( $y$-polarized component) in a 1D system. Horizontal axis: transverse spatial coordinate; vertical axis: time. As time goes on a jump of half a period takes place in the position of the fluctuating stripes in the left side of the figure, as evidenced by the white frames. Parameters are $E_{0}=0.919, \theta=1$. The integration time is $K t=300$ (60000 integration time steps) and the transverse system size is $L=256 \times \Delta x$.

vector $\nabla \psi_{i}^{0}(\vec{x})$ are eigenfunctions of $M$ with zero eigenvalue. For example, in $D=2$ these two eigenfunctions are $\left(\partial_{x} \psi_{1}^{0}, \ldots, \partial_{x} \psi_{N}^{0}\right)$ and $\left(\partial_{y} \psi_{1}^{0}, \ldots, \partial_{y} \psi_{N}^{0}\right)$. These neutrally stable modes of the linearized dynamics are the Goldstone modes. When noise is present, fluctuations around a stable stationary solution are damped, but Goldstone modes are excited without cost and they dominate the spatial structure of the fluctuations. For a stripe pattern the Goldstone mode is given by the spatial gradient of the stripe pattern. This is another stripe pattern shifted in space by a quarter of the spatial period. The Goldstone mode is associated with rigid spatial displacements of the stationary pattern in the direction perpendicular to the stripes. Such rigid displacements are generated by fluctuations that change the value of $\vec{x}_{0}$, that is, homogeneous fluctuations in a global and arbitrary phase of the stripe pattern. The spatial structure of the fluctuations observed in Fig. 2 reflects a maximum of fluctuations corresponding to the Goldstone mode.

The stripe pattern of the fluctuations is shifted with respect to the underlying steady-state stripe pattern a quarter of the spatial period either to the right or to the left. Over large time scales, fluctuations in a region of the plane can in principle make a half-period spontaneous jump between those two configurations. This implies a spontaneous change in the direction of displacement of the steady-state pattern, to which fluctuations are associated. We have visualized this effect in a one-dimensional (1D) system, described by the same dynamics. The 1D stationary pattern shows a regular modulation along the spatial coordinate, similar to a section of the two-dimensional pattern perpendicular to the direction of the stripes. Again, the spatial structure of the fluctuations 


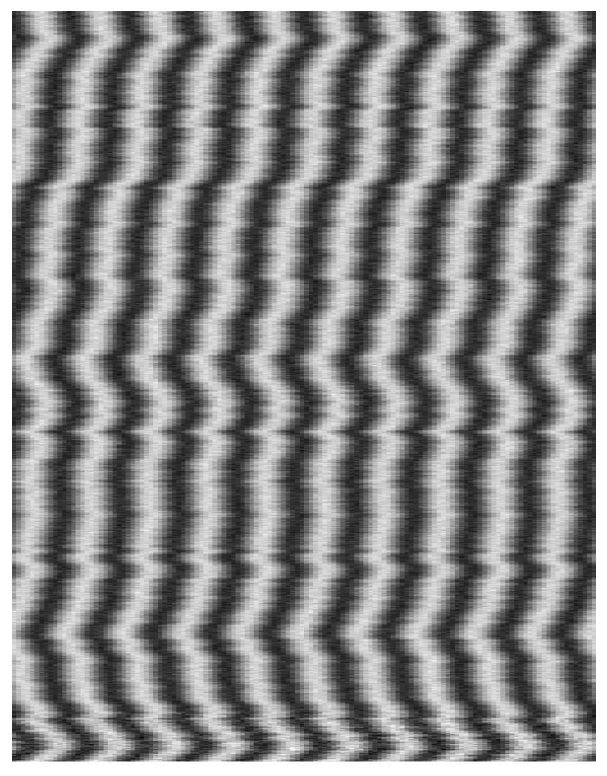

FIG. 4. Spatiotemporal evolution of the real part of the $y$ polarized component of the field, using the nonlinear Langevin equations (10) in a 1D system. Horizontal axis: spatial transverse coordinate; vertical axis: time. We show 2000 snapshots of the transverse field distribution, taken every 400 time units (80000 integration time steps), in a lattice of 128 points. The stripe on the bottom shows the time-averaged pattern. The noise strength $\left(2 n_{s}\right)^{-1 / 2}$ is 0.02 , and the other parameters are as in Fig. 2.

reflects the corresponding Goldstone mode with right or left displacements of the stationary pattern. We have plotted the time evolution of the spatial distribution of fluctuations in Fig. 3. In this figure the two white frames evidence a portion of the transverse section of the beam where, as time goes on, a phase jump takes place.

The random rigid motion of the stripe pattern in the $1 \mathrm{D}$ case can be shown explicitly by displaying the entire field instead of the fluctuations of the field. This can be obtained by numerically integrating the full nonlinear Langevin equations (10) for the field [34]. Figure 4 shows a stochastic realization, from which it can be clearly seen that the location of the maxima and minima of the pattern move in space as time goes on. This is precisely the diffusive motion of the roll pattern predicted in Ref. [5]. The stripe on the bottom of Fig. 4 shows the time average of the pattern, which confirms that the roll pattern is washed out by this motion, if the average is made over time intervals large with respect to the diffusion time. Figure 4 confirms also that the fluctuations associated with the Goldstone mode, and leading to rigid spatial displacements to the left and to the right randomly, are undamped. Being more precise, the motion of the patterns is not strictly rigid. The reason for this is that in a large continuous system there are long-wavelength and weakly damped modes, connected with the Goldstone mode, that are easily excited by noise (soft modes) [33]. These continuous band of modes do not come into play when a continuous system is described in an approximation consisting of a few discrete modes. These soft modes are responsible for the local deformations of the fluctuating pattern. They are also known to destroy long-range order in 1D systems in the limit of systems of infinite size [35]. An example of this decaying correlations in a prototype model (Swift-Hohenberg equations) of pattern formation is discussed in Ref. [36]. Our numerical simulations are made for rather large optical systems. Still they are far from the limit of infinite size considered from a statistical physics point of view. In order to see how long-range order is destroyed, we need to consider a system with a size $L$ much larger than the correlation length l. To visualize this effect, we show in Fig. 5 the result of a simulation for a system which is 64 times larger than the one of Fig. 4. One observes domains of the system with a size given by the correlation length, $l \ll L$, in which the pattern drifts as a whole in a given direction, as it was the case in Fig. 4. However, the pattern moves locally in different directions giving a local drifting in opposite directions for different regions of the system. The pattern is essentially coherent in domains of the size of $l$, but there is no long-range order in the system as a whole.

In $2 \mathrm{D}$, and for relatively small systems, we observe stripes that can be understood as a set of strongly coupled 1D patterns. A zero wave number in the direction of the stripes is strongly dominant. The field fluctuations are still governed and patterned by the Goldstone mode as shown in Fig. 2. However, phase jumps as the one described in Fig. 3 are very unlikely during finite observation times. In addition, the long-wavelength soft modes associated with the Goldstone
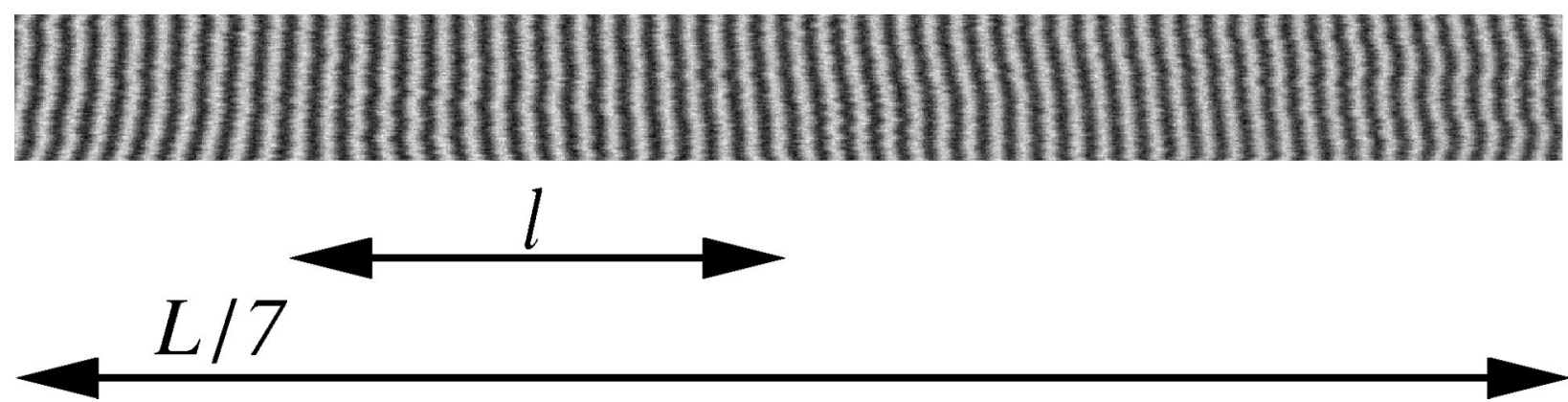

FIG. 5. Spatiotemporal evolution of the real part of the $y$ polarized component of the field, in a large 1D system. The total system size is $L=8192 \times \Delta x$ and the total integration time is 10000 . Only one seventh of the total system is shown here. The correlation length of the pattern $l$ is indicated. Other parameters are as in Fig. 4. 
modes are known to destroy long-range order in spatial dimension larger than 2 . When excited by noise they soften the order parameter, but while long-range order is completely lost in $d=1, d=2$ is the critical dimension with a logarithmic divergence such that the periodicity is not totally lost [35].

\section{FIELDS OUTSIDE THE CAVITY}

In the framework of standard input-output formalism for optical cavities [37], the field immediately outside the cavity coupling mirror $A_{ \pm}^{\text {out }}$ is linked to the intracavity field, and to the reflected input field $A_{ \pm}^{\text {in }}$ by

$$
A_{ \pm}^{\text {out }}(\vec{x}, t)=\sqrt{2} A_{ \pm}(\vec{x}, t)-A_{ \pm}^{\text {in }}(\vec{x}, t),
$$

where scaling [see relations (11)] is such that $\left\langle A_{ \pm}^{\text {out } \dagger} A_{ \pm}^{\text {out }}\right\rangle\left(\left\langle A_{ \pm}^{\text {in } \dagger} A_{ \pm}^{\text {in }}\right\rangle\right)$ represents the average number of photons crossing an area $a$ in a time $K^{-1}$. Notice that the mean value of the input field is related to the pump amplitude introduced in Eq. (4) by $\left\langle A_{ \pm}^{i n}\right\rangle=E_{0} / \sqrt{2}$. Hence, taking the mean value of Eq. (25) we have

$$
F_{ \pm}^{\text {out }}(\vec{x}, t)=\sqrt{2} F_{ \pm}(\vec{x}, t)-E_{0} / \sqrt{2}
$$

The same input-output relation (25) holds for the field fluctuation operators $\delta A_{ \pm}$.

In the quasiclassical description of quantum fluctuations [29], also $c$-number fluctuations $\Delta \alpha_{ \pm}(\vec{x}, t)$ in the Wigner representation have similar input-output relations:

$$
\Delta \alpha_{ \pm}^{\text {out }}(\vec{x}, t)=\sqrt{2} \Delta \alpha_{ \pm}(\vec{x}, t)-\Delta \alpha_{ \pm}^{\text {in }}(\vec{x}, t)
$$

where now $\Delta \alpha_{ \pm}^{i n}(\vec{x}, t)$ are Gaussian stochastic processes that represent input vacuum fluctuations, and have correlations given by Eq. (20).

Intracavity fluctuations $\Delta \alpha_{ \pm}(\vec{x}, t)$ can be simulated by means of numerical integration of Eq. (19). The problem arises with the input term $\Delta \alpha_{i}^{i n}(\vec{x}, t)$, because it is a Gaussian white noise, $\delta$ correlated in time, so that its instantaneous value is ill defined. This implies that the instantaneous value of the input and output fields is ill defined. A similar problem is encountered when calculating the instantaneous frequency during the switch on of a laser using a semiclassical model that includes spontaneous emission white noise [38]. A way to give meaning to these fast fluctuating quantities is to average the fluctuations integrating in a time window $[38,39]$. In numerical simulations, the divergence is strictly avoided due to the fact that the time is discretized. However, it is usually necessary to take very small time steps to preserve the accuracy, so that large fluctuations will still be present. This behavior can be regularized taking the average over several steps of integration that corresponds precisely to the integration of fluctuations in a time window.

We briefly discuss here how to perform a numerical realization of the output field. This requires the integration of Eq. (19) together with Eq. (27). We consider a discretized
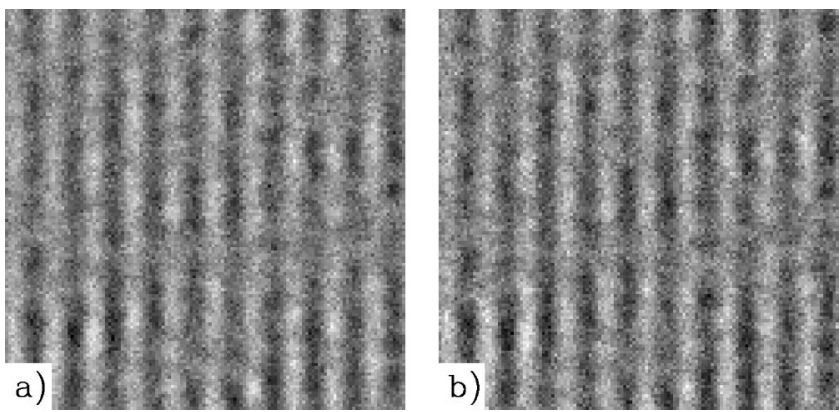

FIG. 6. (a) Distribution in the transverse plane of the $y$-polarized output field fluctuations (real part). The output field has been averaged over 0.5 time units, which corresponds to 100 integration time steps. For comparison, the instantaneous real part of the fluctuations of the field inside the cavity is shown in (b). Parameters as in Fig. 2.

time $t=n \tau$, where $\tau \ll 1$ is the time step for integration. From Eq. (27), the output field integrated over one time step, is

$$
\begin{aligned}
\Delta \alpha_{ \pm, n}^{\text {out }}(\vec{x}) & \cong \int_{n \tau}^{(n+1) \tau} \Delta \alpha_{ \pm}^{\text {out }}(\vec{x}, t) d t \\
& =\sqrt{2} \int_{n \tau}^{(n+1) \tau} \Delta \alpha_{ \pm}(\vec{x}, t) d t-\Delta \alpha_{ \pm, n}^{\text {in }}(\vec{x}),
\end{aligned}
$$

where $\Delta \alpha_{ \pm, n}^{i n}(\vec{x})$ are the input field fluctuations, integrated over one time step:

$$
\Delta \alpha_{ \pm, n}^{i n}(\vec{x})=\int_{n \tau}^{(n+1) \tau} \Delta \alpha_{ \pm}^{i n}(\vec{x}, t) d t
$$

These are Gaussian random numbers of zero mean and correlations

$$
\begin{gathered}
\left\langle\Delta \alpha_{i, n}^{i n}(\vec{x}) \Delta \alpha_{j, n^{\prime}}^{* i n}\left(\vec{x}^{\prime}\right)\right\rangle=\frac{\tau}{2} \delta_{i, j} \delta\left(\vec{x}-\vec{x}^{\prime}\right) \delta_{n n^{\prime}}, \\
(i, j)=(+,-)
\end{gathered}
$$

From Eq. (19) we have

$$
\begin{aligned}
\Delta \alpha_{ \pm}(\vec{x}, n \tau+s)= & \Delta \alpha_{ \pm}(\vec{x}, n \tau)+Q_{ \pm}(n \tau) s \\
& +\sqrt{2} \int_{n \tau}^{n \tau+s} \Delta \alpha_{ \pm}^{i n}\left(\vec{x}, t^{\prime}\right) d t^{\prime}+\mathcal{O}\left(s^{3 / 2}\right),
\end{aligned}
$$

where $Q_{+}\left(Q_{-}\right)$correspond to the expressions $Q_{1}\left(Q_{3}\right)$ given in Appendix B. Then, integrating both sides of Eq. (31) over a time step:

$$
\begin{aligned}
\int_{n \tau}^{(n+1) \tau} \Delta \alpha_{ \pm}(\vec{x}, t) d t \approx & \Delta \alpha_{ \pm}(\vec{x}, n \tau) \tau+\frac{\tau^{2}}{2} Q_{ \pm}(n \tau) \\
& +\sqrt{2} \int_{n \tau}^{(n+1) \tau} d t \int_{n \tau}^{t} \Delta \alpha_{ \pm}^{i n}\left(\vec{x}, t^{\prime}\right) d t^{\prime}
\end{aligned}
$$


Substituting Eq. (32) in Eq. (28), we have

$$
\frac{\Delta \alpha_{ \pm, n}^{\text {out }}(\vec{x})}{\tau}=\sqrt{2} \Delta \alpha_{ \pm, n}(\vec{x})+\frac{\tau}{\sqrt{2}} Q_{ \pm}(n \tau)+\frac{\eta_{ \pm, n}(\vec{x})}{\tau}
$$

where terms of order higher than $\tau$ have been neglected. $\eta_{ \pm, n}(\vec{x})$ are defined as

$$
\eta_{ \pm, n}(\vec{x})=2 \int_{n \tau}^{(n+1) \tau} d t \int_{n \tau}^{t} \Delta \alpha_{ \pm}^{i n}\left(\vec{x}, t^{\prime}\right) d t^{\prime}-\Delta \alpha_{ \pm, n}^{i n}(\vec{x}) .
$$

As they are a linear combination of a Gaussian process, $\eta_{ \pm, n}$ are Gaussian random numbers with zero mean and correlations given by

$$
\left\langle\eta_{i, n}(\vec{x}) \eta_{j, n^{\prime}}^{*}\left(\vec{x}^{\prime}\right)\right\rangle=\left(\frac{2}{3} \tau^{3}-\tau^{2}+\frac{1}{2} \tau\right) \delta_{i j} \delta\left(\vec{x}-\vec{x}^{\prime}\right) \delta_{n n^{\prime}} .
$$

The correlation between $\eta_{ \pm, n}(\vec{x})$ and the input fluctuations is given by

$$
\left\langle\eta_{i, n}(\vec{x}) \Delta \alpha_{j, n^{\prime}}^{* i n}\left(\vec{x}^{\prime}\right)\right\rangle=\frac{\tau}{2}(\tau-1) \delta_{i j} \delta\left(\vec{x}-\vec{x}^{\prime}\right) \delta_{n n^{\prime}}
$$

In Fig. 6 we show a realization of the output field fluctuations averaged over 100 integration time steps (this is $\left.(1 / 100 \tau) \sum_{n=1}^{100} \Delta \alpha_{n}^{\text {out }}\right)$ which corresponds to a time window of 0.5 time units. The output fluctuations [Fig. 6(a)] are qualitatively very similar to the intracavity fluctuations [Fig. 6(b)].

\section{QUANTUM CORRELATIONS}

Quantum fluctuations in the presence of a stripe pattern in the $y$-polarized field have been already studied in this system using a three-mode approximation [7]. In this section we make a comparison between the results of Ref. [7] and the ones obtained using the continuous model presented in this paper. In particular, we want to check the conditions for using the Kerr cavity as a QND measurement device. We consider an $x$-polarized input field that carries a signal that has to be measured with the smallest possible perturbation. The idea is to take advantage of the correlations between the pattern fluctuations and the homogeneous mode fluctuations [40] to perform an indirect measurement of the signal fluctuations by measuring the fluctuations of the pattern modes $\vec{k}_{c}$ and $-\vec{k}_{c}$ [7], which work as meter in the QND measurement. Here $k_{c}=2 \pi / \lambda_{c}$, where $\lambda_{c}$ is the wavelength that characterizes the roll pattern near the instability threshold, and is given by (22). In the far-field plane of the $y$-polarized field, these two modes give rise to an intensity distribution with two large maxima in symmetrical position. In the following we identify the far-field plane with the Fourier plane $\left(k_{x}, k_{y}\right)[41]$, and thus the field distribution in the far-field plane is given by the spatial Fourier transform of the field immediately outside the cavity:

$$
A_{j}^{\text {out }}(\vec{k}, t)=\int \frac{d^{2} \vec{x}}{2 \pi} e^{-i \vec{k} \cdot \vec{x}} A_{j}^{\text {out }}(\vec{x}, t)
$$

We consider the operator

$$
N_{j R}^{\text {out }}(t)=\int_{R} d^{2} \vec{k} A_{j}^{\dagger o u t}(\vec{k}, t) A_{j}^{\text {out }}(\vec{k}, t),
$$

that represents the number of photons with polarization $j$ per unit time over a region $R$ of the far field. In general the polarization could be circular (right or left) or linear ( $x$ or $y$ ). As we did for the intracavity fields (15), we can separate the stationary mean field from the fluctuations in the outputcavity field,

$$
A_{j}^{\text {out }}(\vec{k}, t)=F_{j}^{\text {out }}(\vec{k})+\delta A_{j}^{\text {out }}(\vec{k}, t),
$$

where $F_{+}^{\text {out }}$ is given by Eq. (26). Neglecting second-order terms in the field fluctuations

$$
\left\langle A_{j}^{\dagger \text { out }} A_{j}^{\text {out }}\right\rangle=F_{j}^{* \text { out }} \delta A_{j}^{\text {out }}+F_{j}^{\text {out }} \delta A_{j}^{\dagger \text { out }}+\delta A_{j}^{\text {out }} \delta A_{j}^{\dagger \text { out }},
$$

we introduce the scaled photon fluctuations as,

$$
\delta N_{j R}^{\text {out }}=\frac{\int_{R} d^{2} \vec{k}\left[F_{j}^{* \text { out }}(\vec{k}) \delta A_{j}^{\text {out }}(\vec{k}, t)+\text { H.c. }\right]}{\sqrt{\int_{R} d^{2} \vec{k}\left|F_{j}^{\text {out }}(\vec{k})\right|^{2}}} .
$$

With this normalization, the squeezing spectra take the value 1 for the shot-noise level.

The far-field intensity distributions are strongly peaked around $\vec{k}=0$ ( $x$-polarized component $)$ and $\vec{k}=\vec{k}_{ \pm c}$ (y-polarized component). We are going to consider three regions $R_{0}, R_{1}$, and $R_{2}$, around the homogeneous mode $\vec{k}=0$ and the two pattern modes $\vec{k}_{c}$ and $-\vec{k}_{c}$, respectively. When the size of each region is on the order of the diffraction length $l_{D}$, the whole peak is enclosed. We consider the $x$-polarized photon fluctuations in the first region $\delta N_{x R_{0}}^{\text {out }}$, which for simplicity we will call $\delta N_{0}^{\text {out }}$. In regions $R_{1}$ and $R_{2}$ we consider the $y$-polarized photon fluctuations $\delta N_{1}^{\text {out }}=\delta N_{y R_{1}}^{\text {out }}$ and $\delta N_{2}^{\text {out }}=\delta N_{y R_{2}}^{\text {out }}$. Finally we also consider the $y$-polarized photon fluctuations in the region $R_{1}$ $+R_{2}, \quad \delta N_{1+2}^{\text {out }}=\delta N_{y R_{1}+R_{2}}^{\text {out }}$. Notice that as $\left|F_{j}^{\text {out }}\left(\vec{k}_{c}\right)\right|^{2}$ $=\left|F_{j}^{\text {out }}\left(-\vec{k}_{c}\right)\right|^{2}$ then $\delta N_{1+2}^{\text {out }}=\delta N_{1}^{\text {out }}+\delta N_{2}^{\text {out }}$. In the QND measurements, $\delta N_{0}^{\text {out }}$ is the outgoing signal while we are going to use $\delta N_{1+2}^{\text {out }}$ as the meter.

The expression of the squeezing spectrum of the fluctuations in any of these regions is $[42,43]$,

$$
S_{i}(\omega)=\left\langle\delta N_{i}^{\text {out }} \delta N_{i}^{\text {out }}\right\rangle_{\omega},
$$

where the notation \langle\rangle$_{\omega}$ means Fourier transform of the symmetrized correlation, and it is defined, for some generic variables $W$ and $Z$, as, 


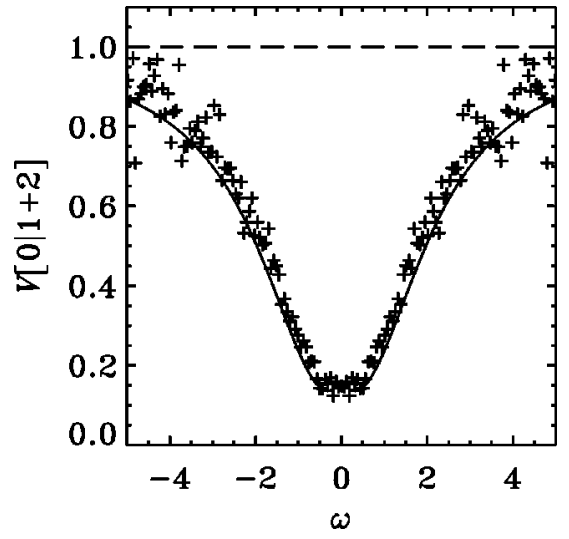

FIG. 7. Conditional variance $V(0 \mid 1+2)$ given by Eq. (45). The solid line represents the three-modes model while the dots represent the continuous model. Parameters: $\eta=-1$ (self-defocusing case), $E_{0}=0.919$, and $\theta=1.7$.

$$
\langle W Z\rangle_{\omega}=\int_{-\infty}^{\infty}\langle W(t) Z(0)\rangle_{s y m m} e^{-i \omega t} d t,
$$

with

$$
\langle W(t) Z(0)\rangle_{\text {symm }}=\langle W(t) Z(0)+Z(0) W(t)\rangle / 2 .
$$

The conditional variance of $N_{0}^{\text {out }}$ given a measurement on $N_{1+2}^{\text {out }}$ is given by,

$$
V(0 \mid 1+2)=S_{0}\left(1-\frac{\left|\left\langle\delta N_{0}^{\text {out }} \delta N_{1+2}^{\text {out }}\right\rangle_{\omega}\right|^{2}}{S_{0} S_{1+2}}\right) .
$$

This is a measure of the correlations between the outgoing signal $N_{0}^{\text {out }}$ and the outgoing meter $N_{1+2}^{\text {out }}$. Strong correlations correspond to small values of $V(0 \mid 1+2)$.

Additionally, we study how the fluctuations of the homogeneous mode are transferred from the input to the output of the cavity (the nondemolition character of the measurement), and also from the input to the pattern modes (accuracy of the measurement). This information is given by the following normalized correlations [42],

$$
\begin{gathered}
C_{s}=\frac{\left|\left\langle\delta N_{0}^{\text {in }} \delta N_{0}^{\text {out }}\right\rangle_{\omega}\right|^{2}}{\left\langle\delta N_{0}^{\text {in }} \delta N_{0}^{\text {in }}\right\rangle_{\omega}\left\langle\delta N_{0}^{\text {out }} \delta N_{0}^{\text {out }}\right\rangle_{\omega}}, \\
C_{m}=\frac{\left|\left\langle\delta N_{0}^{\text {in }} \delta N_{1+2}^{\text {out }}\right\rangle_{\omega}\right|^{2}}{\left\langle\delta N_{0}^{\text {in }} \delta N_{0}^{\text {in }}\right\rangle_{\omega}\left\langle\delta N_{1+2}^{\text {out }} \delta N_{1+2}^{\text {out }}\right\rangle_{\omega}},
\end{gathered}
$$

where the $x$-polarized input fluctuations are given by,

$$
\begin{aligned}
\delta N_{0}^{i n}= & \frac{\int_{R_{0}} d^{2} \vec{k}\left[F_{x}^{* i n}(\vec{k}) \delta A_{x}^{i n}(\vec{k}, t)+\text { H.c. }\right]}{\sqrt{\int_{R_{0}} d^{2} \vec{k}\left|F_{x}^{i n}(\vec{k})\right|^{2}}} \\
= & \delta A_{x}^{i n}(\vec{k}=0, t)+\text { H.c. }
\end{aligned}
$$
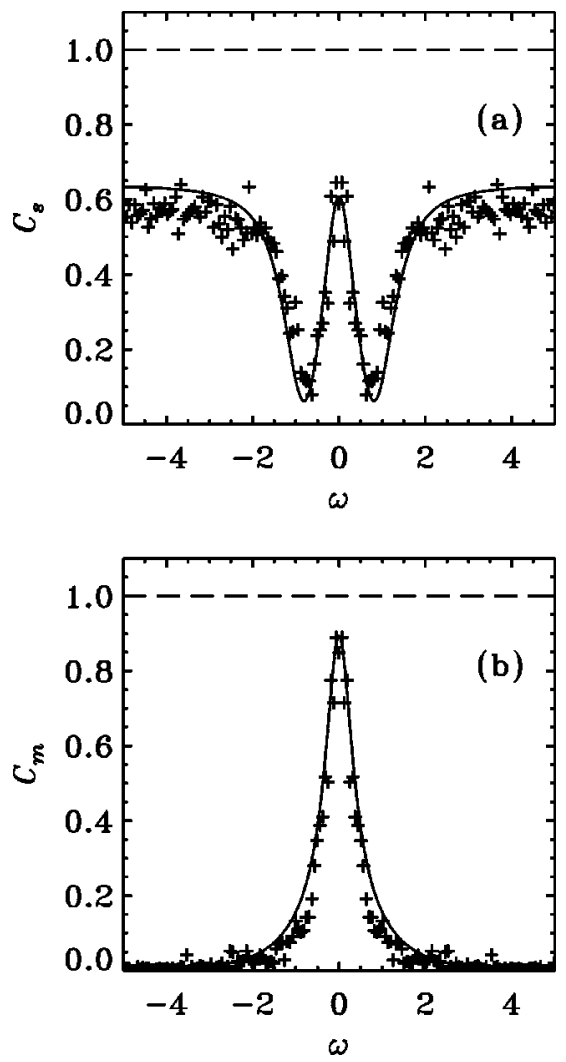

FIG. 8. Correlations $C_{s}$ (a) and $C_{m}$ (b) given by Eq. (47). The solid lines represent the three-modes model while the dots represent the continuous model. Same parameters as in Fig. 7.

The last equality comes from the fact that the input field mean value $F_{x}^{i n}(\vec{k})$ has only contributions at $k=0$. Since the input beam is in a coherent state, the fluctuations correspond to the shot-noise level: $\left\langle\delta N_{0}^{i n} \delta N_{0}^{i n}\right\rangle_{\omega}=1$.

In order to perform a QND measurement of the input field fluctuations $\delta N_{0}^{\text {in }}$ using the pattern fluctuations $\delta N_{1+2}^{\text {out }}$ as a meter, it is required that $V(0 \mid 1+2)<1$ and $C_{s}+C_{m}>1$.

Another quantity of interest (not related to the QND conditions), which is able to show the quantum nature of fluctuations, is the correlation between the two opposite pattern modes $\vec{k}_{c}$ and $-\vec{k}_{c}$. In parametric down conversion it is known that the conservation of transverse momentum leads to the emission of correlated photons that propagate in symmetrical directions; this implies a high correlation between fluctuations in the two symmetric portion of the beam cross section in the far field $[9,10]$. In the process of four wave mixing, present in a $\chi^{(3)}$ medium, the same situation appears. In this case the correlation has been studied with a semiclassical model [40]. The appropriate variable that gives us information about the quantum correlation of the pattern modes is the conditional variance of $N_{1}^{o u t}$ given a measurement on $N_{2}^{\text {out }}$,

$$
V(1 \mid 2)=S_{1}\left(1-\frac{\left|\left\langle\delta N_{1}^{\text {out }} \delta N_{2}^{\text {out }}\right\rangle_{\omega}\right|^{2}}{S_{1} S_{2}}\right) .
$$




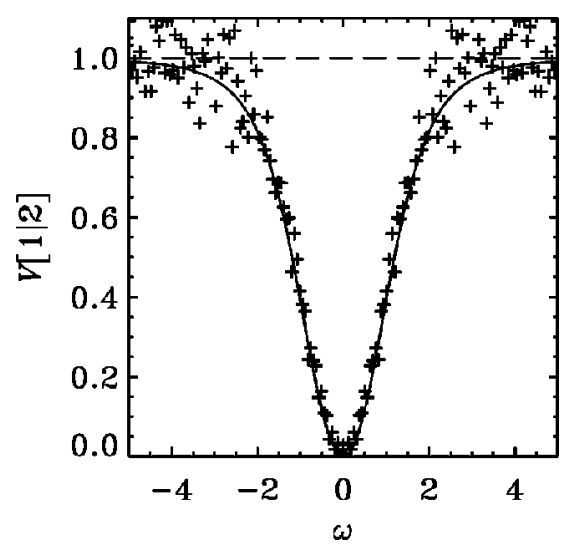

FIG. 9. Conditional variance $V(1 \mid 2)$ given by Eq. (49). The solid line represents the three-modes model while the dots represent the continuous model. Same parameters as in Fig. 7.

If the correlation $\left\langle\delta N_{1}^{\text {out }} \delta N_{2}^{\text {out }}\right\rangle_{\omega}$ is big enough, we will have a variance below the shot-noise level: $V(1 \mid 2)<1$, which means a reduction of the fluctuations below the classical limit.

We have evaluated the correlations and variances described above by numerically simulating Eq. (19) for a detuning $\theta=1.7$. For this value of the detuning, the threshold for pattern formation is $E_{0}^{c}=0.869$. At each time step, the fluctuations in the output field $\Delta \alpha_{ \pm}^{\text {out }}$ have been numerically calculated using Eq. (33) and averaging over a time 0.5, which corresponds to 100 integration time steps. The $c$ numbers corresponding to the operators $\delta N_{R j}^{o u t}$ are calculated as in Eq. (41) substituting $\delta A_{j}^{\text {out }}$ by $\Delta \alpha_{j}^{\text {out }}$. The two times symmetrized correlations (44) are then calculated using the corresponding $c$ numbers and averaging over time for a stochastic realization of the output field. In the results shown below, averages have been performed over 20000 time units. Finally, we fast Fourier transform these correlations to obtain the squeezing spectrum as well as the conditional variances.

In Fig. 7 we show the results for the conditional variance $V(0 \mid 1+2)$ for a pump $E_{0}=0.919$, that is quite close to threshold. The symbols correspond to the results obtained from the continuous model whereas the solid line represents the three-mode approximation [7]. The correlation between the outgoing signal and the outgoing meter is well below the shot-noise level (shown as a dashed line). As predicted by the three-mode model, we can use a vectorial self-defocusing Kerr medium to prepare a state of the homogeneous output with known fluctuations. Comparing both models, the threemode model predicts slightly larger correlations [smaller values for $V(0 \mid 1+2)$ ] than the continuous model. This fact can be explained taking into account that the correlation $\left\langle\delta N_{0}^{\text {out }} \delta N_{1+2}^{\text {out }}\right\rangle_{\omega}$ in Eq. (45) is smaller in the continuous case. This is so because part of the energy is translated to the higher-order modes that were neglected in the three modes approximation.

In Fig. 8 we plot correlations $C_{s}$ and $C_{m}$. As before, the solid line corresponds to results from the three-mode model and the symbols to the continuous model. Again there is a small difference between the two models, which is more clearly seen in the correlation between the incoming and the outgoing signal, $C_{s}$, for high frequencies. Also the peak in the correlation between the incoming signal and the outgoing meter, $C_{m}$, is slightly narrower. Nevertheless, the fitting of the curves is good and demonstrates the validity of the three modes approximation close to the instability threshold. From the plots it can be seen that the condition $C_{s}+C_{m}>1$ is fulfilled for a range of frequencies $|\omega|<0.3$. In this range of frequencies, all the conditions for a QND measurement of the $x$-polarized input fluctuations using the $y$-polarized pattern as a meter, are satisfied.

Similar results can be obtained for other values of the detuning, provided we are below the limit of bistability for the homogeneous solution $(\theta<\sqrt{3})$. However, as the detuning is decreased the QND performance is degraded, as predicted by the three-mode model [7].

Finally, in Fig. 9, we plot the conditional variance $V(1 \mid 2)$. The results of the two models coincide perfectly for small frequencies. As predicted by the three mode model, the conditional variance is dramatically reduced at low frequencies. In fact it goes to zero at zero frequency, showing the existence of strong quantum correlations between the two opposite pattern modes $\vec{k}_{c}$ and $-\vec{k}_{c}$. For larger frequencies, the results form the continuous model shows a large dispersion, although they basically coincide with the prediction from the three mode model. The large dispersion could be reduced increasing the statistics, that is, integrating over a longer stochastic realization.

\section{CONCLUSIONS}

Our analysis, based on the classical-looking set of Langevin equations in the Wigner representation, led to the following main results. The numerical solutions of the complete nonlinear Langevin equations in the 1D spatial case have confirmed that the stripe pattern undergoes locally rigid random translation to the left and to the right, as predicted by the three-mode model [5]. On the other hand, in 2D, phase jumps of the entire pattern are extremely unlikely to occur during finite observation times. The underlying presence of such displacements is manifested, however, by the spatial configuration of the fluctuations, which is dominated by the Goldstone mode. Such a mode is the eigenstate of the linearized problem with zero eigenvalue, and it is given by the spatial gradient of the underlying stationary stripe pattern. The Goldstone mode is always present in a system with broken translational symmetry, as the one that emerges in a pattern forming instability. It is excited, at no cost, by noise and leads to rigid translations of the pattern. In a continuous system there are also soft modes arbitrary close to the Goldstone mode that produce local deformations of the pattern and that would destroy long-range order in 1D systems in the limit of very large system size.

We have formulated a general description of the spatial configuration of the output field immediately beyond the input-output mirror, for an arbitrary size of the time window over which fluctuations are averaged. We have shown that the spatial configuration of the output field is closely similar to that of the intracavity field, provided that this time window is on the order of the cavity linewidth $K^{-1}$, which cor- 
responds to select a spectral bandwidth of the output fluctuations on the same order as that of the intracavity fluctuations.

We analyzed on the one side the anticorrelation between the quantum fluctuations of the intensity of the $x$-polarized pump field and the $y$-polarized pattern modes, and on the other the correlation between the intensity fluctuations of the two symmetrical components (twin beams) of the $y$-polarized field. It turns out that the predictions of the three-mode model are in good agreement with the results of the multimode model. In the case of the system analyzed here, this agreement persists also well beyond the instability threshold in all the regions where the roll pattern is found, because the amplitudes of the modes different from those of the threemode model remain negligibly small. We believe, however, that for other models such an agreement can be found, in general, only close to the instability threshold. Hence the conclusions concerning the validity of the QND scheme formulated in Ref. [7] are confirmed.

\section{ACKNOWLEDGMENTS}

This work is supported by the European Commission through the TMR network QSTRUCT (Project No. FMRXCT96-0077). P. C. and M. S. M. acknowledge financial support from Dirección General de Enseñanza Superior e Investigación Científica (Spain) Project Nos. PB94-1167 and PB97-0141-C02-02. M. H. wants to acknowledge financial support from the FOMEC Project No. 290, Dep. de Física FCEyN, Universidad Nacional de Mar del Plata, and from CONICET Grant No. PIP No. 4342, Argentina. A.G. and L.L. acknowledge the financial support of the MURST project "Spatial pattern control in nonlinear optical systems."

\section{APPENDIX A}

We give here the coefficients of the equation for quasiprobability distribution (9). For simplicity, we use the notation $\left(z_{1}, z_{2}, z_{3}, z_{4}\right)=\left(\alpha_{+}, \alpha_{+}^{*}, \alpha_{-}, \alpha_{-}^{*}\right)$. The dependence on space and time of variables $z_{i}$ is omitted to simplify the notation. The drift terms are,

$$
\begin{gathered}
Q_{1}=\left[-(1+i \eta \theta)+i g \eta(\alpha+\beta / 2)(s-1)+i a \nabla^{2}\right] z_{1} \\
+E_{o}+i \eta g\left[\alpha z_{1} z_{2}+\beta z_{3} z_{4}\right] z_{1}, \\
Q_{2}=Q_{1}^{*} \\
Q_{3}=Q_{3}\left(z_{1}, z_{2}, z_{3}, z_{4}\right)=Q_{1}\left(z_{3}, z_{4}, z_{1}, z_{2}\right), \\
Q_{4}=Q_{3}^{*} .
\end{gathered}
$$

The diffusion terms are,

$$
D_{i j}\left(\vec{x}, \vec{x}^{\prime}\right)=\Gamma_{i j} \delta\left(\vec{x}-\vec{x}^{\prime}\right)
$$

$$
\Gamma=\left(\begin{array}{cccc}
c_{s} \alpha z_{1}^{2} & 1-s & c_{s} \beta z_{1} z_{3} & 0 \\
1-s & -c_{s} \alpha z_{2}^{2} & 0 & -c_{s} \beta z_{2} z_{4} \\
c_{s} \beta z_{1} z_{3} & 0 & c_{s} \alpha z_{3}^{2} & 1-s \\
0 & -c_{s} \beta z_{2} z_{4} & 1-s & -c_{s} \alpha z_{4}^{2}
\end{array}\right)
$$

with $c_{s}=i \eta g s$. The third-order terms are,

$$
T_{i j l}\left(\vec{x}, \vec{x}^{\prime}, \vec{x}^{\prime \prime}\right)=\Theta_{i j l} \delta\left(\vec{x}-\vec{x}^{\prime}\right) \delta\left(\vec{x}^{\prime}-\vec{x}^{\prime \prime}\right),
$$

where the nonzero terms of $\Theta_{i j l}$ are

$$
\begin{gathered}
\Theta_{[112]}=i \frac{\alpha}{2}\left(1-s^{2}\right) \eta g z_{1}, \quad \Theta_{[334]}=i \frac{\alpha}{2}\left(1-s^{2}\right) \eta g z_{3}, \\
\Theta_{[122]}=\Theta_{112}^{*}, \quad \Theta_{[344]}=\Theta_{334}^{*}, \\
\Theta_{[123]}=\frac{\beta}{2 \alpha} \Theta_{334}, \quad \Theta_{[124]}=\frac{\beta}{2 \alpha} \Theta_{344}, \\
\Theta_{[234]}=\frac{\beta}{2 \alpha} \Theta_{122}, \Theta_{[134]}=\frac{\beta}{2 \alpha} \Theta_{112} .
\end{gathered}
$$

Square brackets ([]) indicate the possible permutations of indices.

\section{APPENDIX B}

In this appendix we give the coefficients of the FokkerPlanck equation (18). As in Appendix A, we use the notation $\left(z_{1}, z_{2}, z_{3}, z_{4}\right)=\left(\Delta \alpha_{+}, \Delta \alpha_{+}^{*}, \Delta \alpha_{-}, \Delta \alpha_{-}^{*}\right)$. For the drift terms we have,

$$
\begin{aligned}
& Q_{1}=\left\{-(1+i \eta \theta)+i \nabla^{2}+i \eta\left[2 \alpha\left|F_{+}(\vec{x})\right|^{2}\right.\right. \\
&\left.\left.+\beta\left|F_{-}(\vec{x})\right|^{2}\right]\right\} z_{1}(\vec{x}, t)+i \eta\left[\alpha F_{+}^{2}(\vec{x}) z_{2}(\vec{x}, t)\right. \\
&\left.+\beta F_{+}(\vec{x}) F_{-}^{*}(\vec{x}) z_{3}(\vec{x}, t)+\beta F_{+}(\vec{x}) F_{-}(\vec{x}) z_{4}(\vec{x}, t)\right], \\
& Q_{2}=Q_{1}^{*}, \\
& Q_{3}=\left\{-(1+i \eta \theta)+i \nabla^{2}+i \eta\left[2 \alpha\left|F_{-}(\vec{x})\right|^{2}\right.\right. \\
&\left.\left.+\beta\left|F_{+}(\vec{x})\right|^{2}\right]\right\} z_{3}(\vec{x}, t)+i \eta\left[\alpha F_{-}^{2}(\vec{x}) z_{4}(\vec{x}, t)\right. \\
&\left.+\beta F_{-}(\vec{x}) F_{+}^{*}(\vec{x}) z_{1}(\vec{x}, t)+\beta F_{-}(\vec{x}) F_{+}(\vec{x}) z_{2}(\vec{x}, t)\right], \\
& Q_{4}=Q_{3}^{*} .
\end{aligned}
$$

The diffusion terms are

$$
D_{i j}\left(\vec{x}, \vec{x}^{\prime}\right)=\Gamma_{i j} \delta\left(\vec{x}-\vec{x}^{\prime}\right), \quad \Gamma=\left(\begin{array}{cccc}
0 & 1 & 0 & 0 \\
1 & 0 & 0 & 0 \\
0 & 0 & 0 & 1 \\
0 & 0 & 1 & 0
\end{array}\right) .
$$


[1] For a review, see for example (a) L. A. Lugiato, Chaos, Solitons Fractals 4, 1251 (1994), and references quoted therein; (b) L. A. Lugiato, M. Brambilla, and A. Gatti, in Advances in Atomic Molecular and Optical Physics, edited by B. Bederson and H. Walther (Academic Press, New York, 1998).

[2] A. Gatti, L. A. Lugiato, K. I. Petsas, and I. Marzoli, Europhys. Lett. 46, 461 (1999).

[3] L. A. Lugiato, A. Gatti, and H. Wiedemann, in Quantum Fluctuations, edited by S. Reynaud, E. Giacobino, and J. Zinn Justin (Elsevier-North-Holland, Amsterdam, 1997).

[4] L. A. Lugiato and F. Castelli, Phys. Rev. Lett. 68, 3284 (1992).

[5] L. A. Lugiato and G. Grynberg, Europhys. Lett. 29, 675 (1995).

[6] F. Castelli and L. A. Lugiato, J. Mod. Opt. 44, 765 (1997).

[7] M. Hoyuelos, A. Sinatra, P. Colet, L. Lugiato, and M. San Miguel, Phys. Rev. A 59, 1622 (1999).

[8] A. Gatti and L. A. Lugiato, Phys. Rev. A 52, 1675 (1995).

[9] I. Marzoli, A. Gatti, and L. A. Lugiato, Phys. Rev. Lett. 78, 2092 (1997).

[10] A. Gatti, H. Wiedemann, L. A. Lugiato, I. Marzoli, G. L. Oppo, and S. M. Barnett, Phys. Rev. A 56, 877 (1997).

[11] L. A. Lugiato, A. Gatti, H. Ritsch, I. Marzoli, and G. L. Oppo, J. Mod. Opt. 44, 1899 (1997).

[12] C. Szwaj, G.-L. Oppo, A. Gatti, and L. A. Lugiato, Eur. Phys. J. D 10, 433 (2000).

[13] M. I. Kolobov and I. V. Sokolov, Phys. Lett. A 140, 101 (1989)

[14] M. I. Kolobov and I. V. Sokolov, Europhys. Lett. 15, 271 (1991).

[15] M. I. Kolobov, Phys. Rev. A 44, 1986 (1991).

[16] M. I. Kolobov and P. Kumar, Opt. Lett. 18, 849 (1993).

[17] C. Sibilia, V. Schiavone, M. Bertolotti, R. Horak, and I. Perina, J. Opt. Soc. Am. B 11, 2175 (1994).

[18] E. M. Nagasako, R. W. Boyd, and G. S. Agarwal, Phys. Rev. A 55, 1412 (1997).

[19] G. Grynberg and L. A. Lugiato, Opt. Commun. 101, 69 (1993).

[20] J. B. Geddes, J. V. Moloney, E. M. Wright, and W. J. Firth, Opt. Commun. 111, 623 (1994).

[21] M. Hoyuelos, P. Colet, M. San Miguel, and D. Walgraef, Phys. Rev. E 58, 2992 (1998).

[22] L. A. Lugiato and L. Lefever, Phys. Rev. Lett. 58, 2209 (1987).

[23] R. Bonifacio and L. A. Lugiato, Lett. Nuovo Cimento Soc. Ital. Fis. 21, 505 (1978).
[24] R.W. Boyd, Nonlinear Optics (Academic Press, San Diego, 1992).

[25] Notice that the correspondence with the notation used in Refs. $[20,21]$ is $\alpha=A$ and $\beta=A+B$.

[26] C. W. Gardiner, Quantum Noise (Springer, Berlin, 1991).

[27] H. Carmichael, An Open Systems Approach to Quantum Optics, Lecture Notes in Physics, Vol. m18 (Springer, Berlin, 1993).

[28] P. D. Drummond, Phys. Rev. A 33, 4462 (1986).

[29] S. Reynayd, A. Heidmann, E. Giacobino, and C. Fabre, Prog. Opt. 30, 1 (1992).

[30] On top of the homogeneous part, the $x$-polarized component of the stationary solution shows very small amplitude stripes with wave vector $2 k_{c}$ [21].

[31] The numerical scheme used for integration of Eq. (14) was described in detail by R. Montagne, E. Hernández- García, A. Amengual, and M. San Miguel, Phys. Rev. E 56, 151 (1997). The method is pseudospectral and second-order accurate in time, and is similar to the so-called two-step method. Lattices of size $128 \times 128$ were used with $\Delta x=0.6012$ and $d t=0.05$.

[32] We use lattices of the same size as in Ref. [31] but with smaller time steps $d t=0.005$.

[33] D. Foster, Hydrodynamic Fluctuations, Broken Symmetry, and Correlation Fluctuations (Addison Wesley, Redwood City, CA, 1983).

[34] Numerical integration of the nonlinear Langevin equations (10) was performed using the same method than for the linear Langevin equations (19).

[35] S. K. Ma, Statistical Mechanics (World Scientific, Singapore, 1985), Sec. 29.2.

[36] J. Vinyals, E. Hernandez-Garcia, M. San Miguel, and R. Toral, Phys. Rev. A 44, 1123 (1991).

[37] M. J. Collett and C. W. Gardiner, Phys. Rev. A 30, 1386 (1984).

[38] S. Balle, F. De Pasquale, N. B. Abraham, and M. San Miguel, Phys. Rev. A 45, 1955 (1991).

[39] S. Balle, N. B. Abraham, P. Colet, and M. San Miguel, IEEE J. Quantum Electron. 29, 33 (1993).

[40] M. Hoyuelos, P. Colet, and M. San Miguel, Phys. Rev. E 58, 74 (1998).

[41] Strictly speaking the far field of such a system is located at infinite distance, and we suppose to bring it to a finite distance by making use of a lens.

[42] M. J. Holland, M. Collett, D. F. Walls, M. D. Levenson, Phys. Rev. A 42, 2995 (1990).

[43] A. Sinatra, J. F. Roch, K. Vigneron, Ph. Grelu, J. Ph. Poizat, K. Wang, and P. Grangier, Phys. Rev. A 57, 2980 (1998). 